\section{A digitally programmable attenuator}

\author{
RICHARD V. WOLF* \\ Bioacoustic Laboratory \\ Eye and Ear Hospital \\ University of Pittsburgh \\ Pittsburgh, Pennsylvania 15213
}

The simplest and most trouble-free method of digitally controlling the level of low-frequency signals involves mechanically switching fixed attenuator sections (i.e., pads) within the signal chain (Farley, 1971). The pads can be arranged in conveniently sized and well defined steps, using either a binary or binary-coded decimal progression. Several digital attenuators have been built using the circuit and components shown in Fig. 1 to cover a range of $1277 / 8 \mathrm{~dB}$ in steps as small as $1 / 8 \mathrm{~dB}$. The circuit contains no active devices and is constructed entirely from commercially available components. This attenuator has a constant impedance $(600 \mathrm{Ohms})$, regardless of the number or position of the pads in use. It can handle large signals ( $1 \mathrm{~W}$ ) from dc to $20 \mathrm{kHz}$ without distortion and with extremely low noise levels.

The parts list (Table 1) calls for relatively expensive and physically large mercury-wetted relays (MWR) primarily because inconsistent contact resistance can destroy the reliability of circuits containing critically proportioned resistor networks. The MWRs not only minimize contact deterioration over time but also increase operating speed, reduce contact capacitance, and eliminate noise-producing contact bounce. Indicator lights, driven from the third pole of each relay, provide a means of monitoring the state of the attenuator while under computer control. If the logic circuits used to drive the relays can handle the additional current required by the lamps, then two pole relays can be used, with an appreciable reduction in cost. Front panel switches are included so that the attenuator can be set manually to facilitate signal-level measurements.

Although construction of the attenuator is straightforward, certain minimum precautions should be observed. Shielded leads should be used for all signal handling circuits,

*The author wishes to acknowledge Dr. Robert $C$. Bilger, whose suggestions led to the simplified and straightforward approach to the construction of this device.

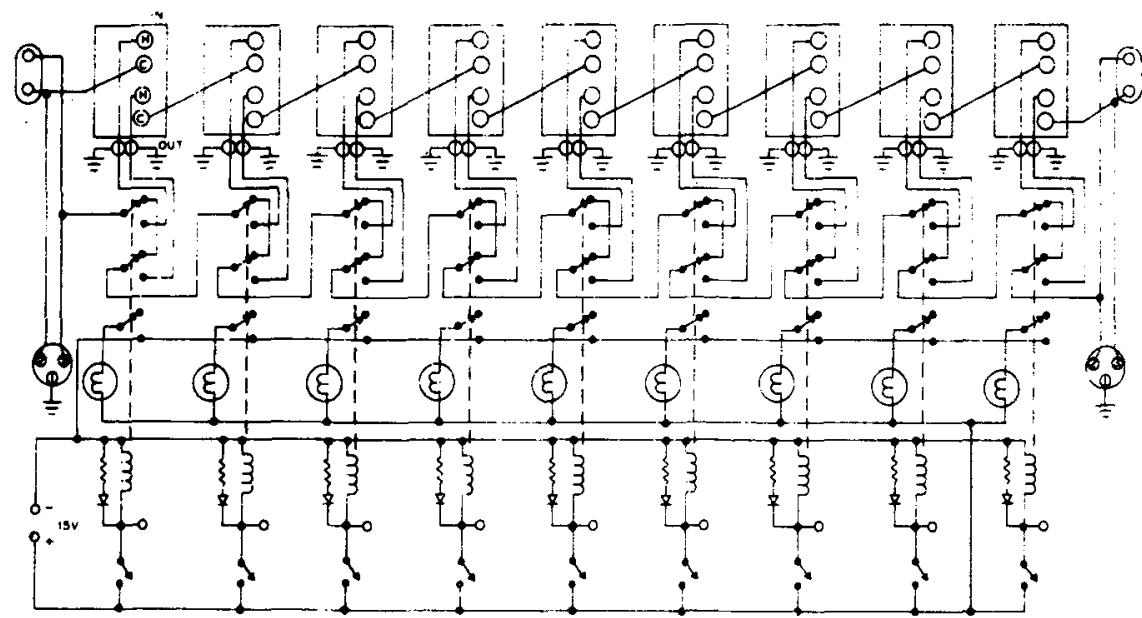

Fig. 1. A 9-bit $637 / 8-\mathrm{dB}$ attenuator schematic. The $G / R$ binding posts are mounted on the front panel to allow in circuit measurement, while the signal is routed through the attenuator with Cannon connectors at the rear. The common lead of each pad should not be grounded to the attenuator chassis except at the input or output. The common leads are not shielded. The power supply and diode/resistor suppression circuits are for negative level drivers.

and the shields should be grounded at each pad. Wherever possible, control wiring should be routed away from the signal circuits. The largest pads should be located near the output of the attenuator chain to minimize power dissipation and resultant resistance change with temperature.

The relays specified in the parts list will operate in less than $5 \mathrm{msec}$ and have make-before-break contacts. This contact arrangement will, under certain conditions, result in zero attenuation and less than 600 Ohms impedance during the 5-msec

Table 1

Parts List for Attenuator Shown in Fig. 1*

9 Each Mercury wetted relays (PotterBrumfield JM3-109-31 or equivalent)

9 Relay sockets

9 28-V lamps and sockets

9 SPST switches

1 Rack panel: $7 \times 19$ in.

1 Chassis $8 \times 17 \times 3$ in. with bottom plate and brackets

IN 4816 diodes

$100 \Omega, 1 / 2-W$ resistors

Dual binding post assemblies

XLR chassis connector, one male. one female

Connector for control line inputs

Fixed loss pads, unbalanced "T" network, 600-Ohms input and out put impedance (Langevin Model FN-100-T or equivalent, 1/8, 1/4, $1 / 2,1,2,4,8,16$, and $32 \mathrm{~dB}$ )

*To extend the attenuation to $127 \mathrm{~dB}$, one more relay controlling 2- to 32-dB pads in series should be added. switching period. If zero attenuation during the switching period is a problem, then attenuation can be changed in stages to avoid switching all in-circuit pads at the same time. The impedance change is unavoidable in mechanically switched circuits and will only present problems when the attenuator is used with minimum loss passive networks (e.g., resistive adders) that require constant impedance at all network terminals.

The 1/8-dB pads are used, not in an attempt to specify this marginally measurable increment, but to provide a means of "benchmark correction" when the attenuator is used in circuits that do not conform to the impedance matching requirements of the device (i.e., $600 \mathrm{Ohms}$ in and out). The least significant bits of our attenuators have $1 / 8-1 / 4-$, and $1 / 2-\mathrm{dB}$ attenuation, follow the binary progression of the rest of the unit, and enable corrections to less than $1 \%$ error for all larger attenuator settings. If this function is not essential, then these sections may be eliminated, further reducing the construction costs.

Although physically large and moderately expensive, these attenuators have provided rapid and reliable control of audio signals in daily use in experiments for more than 3 years.

\section{REFERENCE}

FARLEY, M. F. Digital approach provides precise, programmable AGC. Electronics, $1971,00,52-56$ 